March, 2002

hep-lat/0203019

NIIG-DP-02-2

\title{
Ginsparg-Wilson Relation and Lattice Chiral Symmetry in Fermionic Interacting Theories
}

\author{
Yuji Igarashi ${ }^{a}$, Hiroto So and Naoya Ukita \\ ${ }^{a}$ Faculty of Education, Niigata University, Niigata 950-2181, Japan \\ Department of Physics, Niigata University, Niigata 950-2181, Japan
}

\begin{abstract}
We derive Ginsparg-Wilson relation for a lattice chiral symmetry in theories with self-interacting fermions. Auxiliary scalar and pseudo-scalar fields are introduced on a coarse lattice to give an effective description of the fermionic interactions. We obtain particular solutions to the Ginsparg-Wilson relation and other WardTakahashi identities in a closed form. These non-perturbative solutions can be used to construct a chiral invariant action and an invariant path-integral measure on the coarse lattice. The resulting partition function exhibits the exact chiral symmetry in the fermionic system with the auxiliary fields.
\end{abstract}

PACS: 11.10Hi; 11.15.Tk; 11.30.-j

Keywords: Lattice Quantum Field Theory, Anomalies in Fields and String Theories. 


\section{Introduction}

In the last decade, considerable progress has been made in realization of the chiral symmetry on the lattice. The crucial issue in this realization lies in an algebraic constraint on lattice Dirac operators derived by Ginsparg and Wilson [1]. An explicit solution to the Ginsparg-Wilson (GW) relation [1] was given [2]. It exhibits some remarkable properties

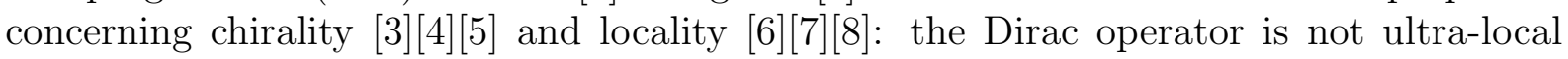
but exponentially local. In the Lüscher's realization of the chiral symmetry [4], the Dirac operator appears in the symmetry transformations, thereby avoiding the no-go theorem [9]. It has been also shown that this formulation of the chiral symmetry correctly gives the index theorem related to the chiral anomaly [3] [1]. See, for example, ref. [10] for reviews of recent development.

The above considerations of the chiral symmetry are based on the GW relation which is derived mainly in free theories. It is highly nontrivial how lattice chiral symmetry can be realized in theories with generic interactions. In order to formulate the symmetry, one has to construct symmetry transformations in such a way that both of action and path-integral measure become chiral invariant. Recently, Lüscher has shown [11] that this can be done in abelian chiral gauge theories with anomaly-free fermion multiplets. This remarkable success is tempting us to formulate lattice chiral symmetry in other interacting theories.

The purpose of this letter is to discuss chiral symmetry in fermionic self-interacting system. To formulate the symmetry, we begin with a microscopic fermionic action on a fine lattice, and then define a macroscopic action on a coarse lattice using the block-spin transformation. The path-integral over the microscopic fields will generate fermionic selfinteraction terms in the macroscopic action. Instead of dealing with such terms directly, we introduce auxiliary fields on the coarse lattice to give an effective description of the fermionic interaction terms. We restrict ourselves here to scalar and pseudo-scalar fields. The lattice action consists of potential of these fields, in addition to fermion bilinear forms with the Yukawa coupling. Our main task is then to derive the Ward-Takahashi (WT) identities which includes the GW relation for this action. The resulting GW relation depends on the auxiliary fields, and gives an extension of the original GW relation in free theories. The relation we obtain can be solved by making suitable locality assumption on the chiral transformations of the auxiliary fields. It is found in our construction that the auxiliary fields are transformed nonlinearly. We determine chiral invariants made of the auxiliary fields, and a counter term needed to cancel the change in the path integral measure defined on the coarse lattice. This can be done by solving the remaining WT identities. The non-perturbative solutions we obtain can be used to define an exact chiral symmetry in quantum system with the auxiliary fields describing the scalar and pseudoscalar couplings of the fermionic self-interactions. Our approach should be compared with those in earlier attempts [4] [12] [13] [14], which were based on the GW relation in free theories.

Since our construction of the symmetry is slightly involved, we present here our main results. A detailed description of the chiral symmetry and its structure will be given 
elsewhere 115.

\section{Macroscopic action with auxiliary fields}

Let us consider a microscopic action $A_{\mathrm{c}}\left[\psi_{\mathrm{c}}, \bar{\psi}_{\mathrm{c}}\right]$ of the Dirac fields $\psi_{\mathrm{c}}(x), \bar{\psi}_{\mathrm{c}}(x)$ carrying a single flavor. These fields are defined on a $d$ (even) dimensional fine-lattice whose positions are labeled by $x$. The microscopic action describes a certain class of fermionic self-interactions specified below. The action is assumed to be invariant under the chiral transformation

$$
\left\{\begin{array}{l}
\psi_{\mathrm{c}}(x) \longrightarrow \psi_{\mathrm{c}}^{\prime}(x) \equiv \psi_{\mathrm{c}}(x)+i \epsilon \gamma_{5} \psi_{\mathrm{c}}(x) \\
\bar{\psi}_{\mathrm{c}}(x) \longrightarrow \bar{\psi}_{\mathrm{c}}^{\prime}(x) \equiv \bar{\psi}_{\mathrm{c}}(x)+\bar{\psi}_{\mathrm{c}}(x) i \epsilon \gamma_{5}
\end{array}\right.
$$

with an infinitesimal constant parameter $\epsilon$ :

$$
A_{\mathrm{c}}\left[\psi_{\mathrm{c}}, \bar{\psi}_{\mathrm{c}}\right] \longrightarrow A_{\mathrm{c}}\left[\psi_{\mathrm{c}}^{\prime}, \bar{\psi}_{\mathrm{c}}^{\prime}\right]=A_{\mathrm{c}}\left[\psi_{\mathrm{c}}, \bar{\psi}_{\mathrm{c}}\right]
$$

Let $A[\Psi, \bar{\Psi}]$ be an effective action of the Dirac fields $\Psi_{n}, \bar{\Psi}_{n}$ defined on a coarse lattice. Indices $n, m$ are used for labeling sites of the lattice. This macroscopic action is obtained from the microscopic action via the block-spin transformation [1]

$$
e^{-A[\Psi, \bar{\Psi}]} \equiv \int \mathcal{D} \psi_{\mathrm{c}} \mathcal{D} \bar{\psi}_{\mathrm{c}} e^{-A_{\mathrm{c}}\left[\psi_{\mathrm{c}}, \bar{\psi}_{\mathrm{c}}\right]-\sum_{n}\left(\bar{\Psi}_{n}-\bar{B}_{n}\right) \alpha\left(\Psi_{n}-B_{n}\right)},
$$

where $\alpha$ is a constant parameter proportional to inverse of the coarse lattice spacing $a$, $\alpha \propto 1 / a$. The gaussian integral in (2.3) relates the macroscopic fields $\Psi_{n}, \bar{\Psi}_{n}$ to the block-spin variables defined by

$$
\left\{\begin{array}{l}
B_{n} \equiv \int d^{d} x f_{n}(x) \psi_{\mathrm{c}}(x) \\
\bar{B}_{n} \equiv \int d^{d} x \bar{\psi}_{\mathrm{c}}(x) f_{n}^{*}(x)
\end{array},\right.
$$

where $f_{n}(x)$ is an appropriate function for coarse graining normalized as $\int d^{d} x f_{n}^{*}(x) f_{m}(x)=$ $\delta_{n m}$.

The path-integral over the microscopic fields in (2.3) will generate fermionic selfinteraction terms in the macroscopic action $A[\Psi, \bar{\Psi}]$. Dealing with such terms directly makes our symmetry analysis complicated. We consider instead an effective theory with some auxiliary fields, where the fermionic interaction terms generated by the block-spin transformation can be replaced by an appropriate function of the auxiliary fields. We introduce auxiliary fields $\sigma_{n}$ and $\pi_{n}$ on the coarse lattice to describe the fermionic scalar and pseudo-scalar interactions. As is well known, these interactions are incorporated into the Nambu-Jona-Lasino model, and recognized as the most important couplings to describe the chiral symmetry and its spontaneous breaking in the effective theory. Therefore, it is reasonable to consider these auxiliary fields, and inclusion of other fields can be done in a similar manner.

The macroscopic action we consider then takes of the form

$$
A[\Psi, \bar{\Psi}]=\Sigma_{n m} \bar{\Psi}_{n}\left(D_{0}\right)_{n m} \Psi_{m}+V\left[\bar{\Psi}_{n}\left(\delta_{n m}+h(\nabla)_{n m}\right) \Psi_{m}, \bar{\Psi}_{n} \gamma_{5}\left(\delta_{n m}+h(\nabla)_{n m}\right) \Psi_{m}\right],
$$


where $D_{0}$ is the Dirac operator for the kinetic term, and $V$ denotes fermionic interactions which consist of contact terms as well as non-contact ones with the difference operators $h(\nabla)_{n m}$. We may obtain the action (2.5) by performing integration over the auxiliary fields in a new macroscopic action:

$$
e^{-A[\Psi, \bar{\Psi}]} \equiv \int \mathcal{D} \pi \mathcal{D} \sigma e^{-\sum_{n m} \bar{\Psi}_{n}\left(\left(D_{0}\right)_{n m}+(\delta+h(\nabla))_{n m}\left(i \gamma_{5} \pi+\sigma\right)_{m}\right) \Psi_{m}-A_{X}[\pi, \sigma]}
$$

It is noted that the Dirac fields appear only bilinearly in the new action. All the fermionic interactions are cast into the Yukawa couplings with the auxiliary fields and the potential term $A_{X}[\pi, \sigma]$. The new action given above can be used to construct an exact chiral symmetry in the macroscopic theory.

\section{Ginsparg-Wilson relation in fermionic interacting theory}

We derive now GW relation for a chiral symmetry in the fermionic interacting theory on the coarse lattice. Our derivation is based on the path-integral relation obtained above:

$$
\begin{aligned}
\int \mathcal{D} \pi \mathcal{D} \sigma e^{-\sum_{n m} \bar{\Psi}_{n} \tilde{D}(\pi, \sigma)_{n m} \Psi_{m}-A_{X}[\pi, \sigma]} \\
=\int \mathcal{D} \psi_{\mathrm{c}} \mathcal{D} \bar{\psi}_{\mathrm{c}} e^{-A_{\mathrm{c}}\left[\psi_{\mathrm{c}}, \bar{\psi}_{\mathrm{c}}\right]-\sum_{n}\left(\bar{\Psi}_{n}-\bar{B}_{n}\right) \alpha\left(\Psi_{n}-B_{n}\right)},
\end{aligned}
$$

where the total Dirac operator is given by

$$
\begin{aligned}
\tilde{D}(\pi, \sigma)_{n m} & =\left(D_{0}\right)_{n m}+(\delta+h(\nabla))_{n m}\left(i \gamma_{5} \pi+\sigma\right)_{m} \\
& \equiv D(\pi, \sigma)_{n m}+\delta_{n m}\left(i \gamma_{5} \pi+\sigma\right)_{m} .
\end{aligned}
$$

It is assumed that the $\tilde{D}(\pi, \sigma)$ is at most linear in $\pi$ and $\sigma$.

In oder to obtain the GW relation as a WT identity, we make a change of variables in both sides of the path-integral. For the microscopic fields $\psi_{c}(x), \bar{\psi}_{c}(x)$, we use the chiral transformation given in (2.1) to have new variables. They lead to new block-spin variables,

$$
\left\{\begin{array}{c}
B_{n}^{\prime} \equiv B_{n}+i \epsilon \gamma_{5} B_{n} \\
\bar{B}_{n}^{\prime} \equiv \bar{B}_{n}+\bar{B}_{n} i \epsilon \gamma_{5}
\end{array} .\right.
$$

For the macroscopic fields $\Psi_{n}, \bar{\Psi}_{n}$, it is reasonable to consider

$$
\left\{\begin{array}{c}
\Psi_{n}^{\prime} \equiv \Psi_{n}+i \epsilon \gamma_{5} \Psi_{n} \\
\bar{\Psi}_{n}^{\prime} \equiv \bar{\Psi}_{n}+\bar{\Psi}_{n} i \epsilon \gamma_{5}
\end{array} .\right.
$$

We may write the transformation on the auxiliary fields as

$$
\left\{\begin{array}{c}
\pi_{n}^{\prime} \equiv \pi_{n}+\epsilon \delta \pi_{n} \\
\sigma_{n}^{\prime} \equiv \sigma_{n}+\epsilon \delta \sigma_{n}
\end{array} .\right.
$$


where $\delta \pi_{n}$ and $\delta \sigma_{n}$ are unknown, and to be determined below. For the Dirac operator $\tilde{D}(\pi, \sigma)$, we have

$$
\tilde{D}^{\prime} \equiv \tilde{D}+\epsilon \delta D+\epsilon\left(i \gamma_{5} \delta \pi+\delta \sigma\right)
$$

Invariance of the path-integral under the above change of variables gives the WT identity for our interacting system. It is derives as follows. Let us make the change of variables given in (2.1), (3.3), (3.4) and (3.5). Then, the l.h.s of (3.1) expressed by new variables turns to be

$$
\begin{aligned}
\int \mathcal{D} \pi^{\prime} & \mathcal{D} \sigma^{\prime} e^{-\sum_{n m} \bar{\Psi}_{n}^{\prime} \tilde{D}\left(\pi^{\prime}, \sigma^{\prime}\right)_{n m} \Psi_{m}^{\prime}-A_{X}\left[\pi^{\prime}, \sigma^{\prime}\right]} \\
= & \int \mathcal{D} \pi \mathcal{D} \sigma \\
\times & {\left[1+\epsilon\left(\delta J_{X}-\delta A_{X}\right)-\epsilon \sum_{n m} \bar{\Psi}_{n}\left(i\left\{\gamma_{5}, \tilde{D}\right\}+\delta \tilde{D}\right)_{n m} \Psi_{m}+\mathcal{O}\left(\epsilon^{2}\right)\right] } \\
\times & \exp \left(-\sum_{n m} \bar{\Psi}_{n} \tilde{D}_{n m} \Psi_{m}-A_{X}\right),
\end{aligned}
$$

where $\delta J_{X}$ denotes the change of $\mathcal{D} \pi \mathcal{D} \sigma . \delta A_{X}$ as well as $\delta \tilde{D}$ are induced by $\delta \pi$ and $\delta \sigma$. On the other hand, the r.h.s of (3.1) turns to be

$$
\begin{aligned}
& \int \mathcal{D} \psi_{\mathrm{c}}^{\prime} \mathcal{D} \bar{\psi}_{\mathrm{c}}^{\prime} e^{-A_{\mathrm{c}}\left[\psi_{\mathrm{c}}^{\prime}, \bar{\psi}_{\mathrm{c}}^{\prime}\right]-\sum_{n}\left(\bar{\Psi}_{n}^{\prime}-\bar{B}_{n}^{\prime}\right) \alpha\left(\Psi_{n}^{\prime}-B_{n}^{\prime}\right)} \\
& =\int \mathcal{D} \psi_{\mathrm{c}} \mathcal{D} \bar{\psi}_{\mathrm{c}} \\
& \times\left[1+\epsilon\left(\delta J_{\mathrm{c}}-\delta A_{c}-\sum_{n}\left(\bar{\Psi}_{n}-\bar{B}_{n}\right) 2 i \alpha \gamma_{5}\left(\Psi_{n}-B_{n}\right)\right)+\mathcal{O}\left(\epsilon^{2}\right)\right] \\
& \times \exp \left(-A_{\mathrm{c}}\left[\psi_{\mathrm{c}}, \bar{\psi}_{\mathrm{c}}\right]-\sum_{n}\left(\bar{\Psi}_{n}-\bar{B}_{n}\right) \alpha\left(\Psi_{n}-B_{n}\right)\right) \\
& =\int \mathcal{D} \psi_{\mathrm{c}} \mathcal{D} \bar{\psi}_{\mathrm{c}} \\
& \times\left[1+\epsilon\left(\delta J_{\mathrm{c}}-\delta A_{c}\right)+\epsilon \sum_{n}\left(\frac{2 i}{\alpha} \frac{\partial^{l}}{\partial \Psi_{n}} \gamma_{5} \frac{\partial^{l}}{\partial \bar{\Psi}_{n}}+2 i \operatorname{Tr} \gamma_{5}\right)+\mathcal{O}\left(\epsilon^{2}\right)\right] \\
& \times \exp \left(-A_{\mathrm{c}}\left[\psi_{\mathrm{c}}, \bar{\psi}_{\mathrm{c}}\right]-\sum_{n}\left(\bar{\Psi}_{n}-\bar{B}_{n}\right) \alpha\left(\Psi_{n}-B_{n}\right)\right)
\end{aligned}
$$

where $\delta J_{\mathrm{c}}$ originates from the change of $\mathcal{D} \psi_{\mathrm{c}} \mathcal{D} \bar{\psi}_{\mathrm{c}}$. The third term with square brackets in the last expression of (3.8) can be placed outside of the integral over the microscopic variables. It leads to

$$
\epsilon \sum_{n}\left(\frac{2 i}{\alpha} \frac{\partial^{l}}{\partial \Psi_{n}} \gamma_{5} \frac{\partial^{l}}{\partial \bar{\Psi}_{n}}+2 i \operatorname{Tr} \gamma_{5}\right) \int \mathcal{D} \pi \mathcal{D} \sigma e^{-\sum_{n m} \bar{\Psi}_{n} \tilde{D}(\pi, \sigma)_{n m} \Psi_{m}-A_{X}[\pi, \sigma]}
$$




$$
\begin{aligned}
= & \int \mathcal{D} \pi \mathcal{D} \sigma \epsilon\left[2 i \sum_{n} \operatorname{Tr}\left(\gamma_{5}-\gamma_{5} \frac{\tilde{D}}{\alpha}\right)_{n n}-\sum_{n m} \frac{2 i}{\alpha} \bar{\Psi}_{n}\left(\tilde{D} \gamma_{5} \tilde{D}\right)_{n m} \Psi_{m}\right] \\
& \times \exp \left(-\sum_{n m} \bar{\Psi}_{n} \tilde{D}(\pi, \sigma)_{n m} \Psi_{m}-A_{X}[\pi, \sigma]\right) .
\end{aligned}
$$

Collecting the contributions of order $\mathcal{O}(\epsilon)$ in (3.7) and (3.8), we obtain

$$
\begin{gathered}
\int \mathcal{D} \psi_{\mathrm{c}} \mathcal{D} \bar{\psi}_{\mathrm{c}}\left(\delta J_{\mathrm{c}}-\delta A_{c}\right) e^{-A_{\mathrm{c}}\left[\psi_{\mathrm{c}}, \bar{\psi}_{\mathrm{c}}\right]-\sum_{n}\left(\bar{\Psi}_{n}-\bar{B}_{n}\right) \alpha\left(\Psi_{n}-B_{n}\right)} \\
=\int \mathcal{D} \pi \mathcal{D} \sigma\left[\left(\delta J_{X}-\delta A_{X}\right)-2 i \sum_{n} \operatorname{Tr}\left(\gamma_{5}-\gamma_{5} \frac{\tilde{D}}{\alpha}\right)_{n n}\right. \\
\left.\quad-\sum_{n m} \bar{\Psi}_{n}\left(i\left\{\gamma_{5}, \tilde{D}\right\}+\delta \tilde{D}-\frac{2 i}{\alpha} \tilde{D} \gamma_{5} \tilde{D}\right)_{n m} \Psi_{m}\right] \\
\times \exp \left(-\sum_{n m} \bar{\Psi}_{n} \tilde{D}(\pi, \sigma)_{n m} \Psi_{m}-A_{X}[\pi, \sigma]\right) .
\end{gathered}
$$

It should be noted that since one introduces the auxiliary fields only on the coarse lattice, one has to perform their integration in the macroscopic action.

For the l.h.s. of (3.10), we may take $\delta A_{c}=\delta J_{c}=0$. The former follows from our requirement of (2.2). The latter, the condition of absence of anomaly in the microscopic theory, is expected to be the case because the theory has no gauge fields. Turn to the r.h.s. of (3.10), therefore, the path-integral over the auxiliary fields gives zero. This allows a wide class of solutions for which the integrand becomes $\pi$ or $\sigma$ derivative of something. We consider here more restrict class of solutions for which the integrand itself vanishes. In order to further reduce this condition, we assume that $\delta \pi_{n}$ and $\delta \sigma_{n}$ are local and do not depend on the fermionic fields $\Psi_{n}, \bar{\Psi}_{n}, B_{n}$ and $\bar{B}_{n}$ : They are functions only of $\pi_{n}$ and $\sigma_{n}$. We thus obtain three conditions,

$$
\begin{gathered}
\bar{\Psi}_{n}\left[\left\{\gamma_{5}, \tilde{D}\right\}-\frac{2}{\alpha} \tilde{D} \gamma_{5} \tilde{D}-i \delta \tilde{D}\right]_{n m} \Psi_{m}=0 \\
\delta A_{X}^{(0)}=0 \\
2 i \sum_{n} \operatorname{Tr}\left(\gamma_{5}-\gamma_{5} \frac{\tilde{D}}{\alpha}\right)_{n n}-\delta J_{X}+\delta A_{X}^{\text {count }}=0 .
\end{gathered}
$$

Here $A_{X}$ is divided into two parts, $A_{X}=A_{X}^{(0)}+A_{X}^{\text {count }}: A_{X}^{(0)}$ denotes a chiral invariant action, and $A_{X}^{\text {count }}$ a counter action.

The first eq. (3.11a) is a generalization of the GW relation for free theory. It depends on the auxiliary fields and tells us how to construct an exact chiral symmetry in the macroscopic theory. The second eq.(3.11b) can be used to fix an invariant potential of the auxiliary fields. The third eq.(3.11) represents the anomaly matching relation: Because of the absence of anomaly in the microscopic theory, the macroscopic theory should be 
anomaly free. The first term in the l.h.s of (3.11d) can be interpreted as the change of the macroscopic path integral measure $\mathcal{D} \Psi \mathcal{D} \bar{\Psi}$ under the chiral transformations constructed below. The second term $\delta J_{X}$ is the change of the macroscopic path integral measure of the auxiliary fields $\mathcal{D} \pi \mathcal{D} \sigma$ under (3.5). If these two contributions do not vanish, they must be eliminated by the contributions from the counter term $\delta A_{X}^{\text {count }}$.

\section{Solutions to the GW relation and other WT iden- tities}

Let us consider first the GW relation (3.11a) which reduces to

$$
\left[\left\{\gamma_{5}, \tilde{D}\right\}-\frac{2}{\alpha} \tilde{D} \gamma_{5} \tilde{D}-i \delta \tilde{D}\right]_{n m}=0
$$

where $\tilde{D}_{n m}=D_{n m}+\delta_{n m} X_{n}$ with $X_{n}=\left(i \gamma_{5} \pi+\sigma\right)_{n}$. We use here again the locality assumption that $\delta X_{n}$ should be only a function of $X_{n}$. Then, the GW relation (4.1) can be decomposed into contact terms and non-contact terms:

$$
\begin{aligned}
& \left\{\gamma_{5}, X_{n}\right\}-\frac{2}{\alpha} X_{n} \gamma_{5} X_{n}-i \delta X_{n}=0 \\
& {\left[\left\{\gamma_{5}, D\right\}-\frac{2}{\alpha} D \gamma_{5} D-\frac{2}{\alpha} D \gamma_{5} X-\frac{2}{\alpha} X \gamma_{5} D-i \delta D\right]_{n m}=0}
\end{aligned}
$$

Since the $X$ commutes with $\gamma_{5}^{\text {日, }}$, it immediately follows from (4.2) that

$$
\left\{\begin{array}{rl}
\delta X_{n} & =-2 i \gamma_{5} X_{n}\left(1-\frac{1}{\alpha} X_{n}\right) \\
\delta \pi_{n} & =-2 \sigma_{n}+\frac{2}{\alpha}\left(\sigma_{n}^{2}-\pi_{n}^{2}\right) \\
\delta \sigma_{n} & =2 \pi_{n}-\frac{4}{\alpha} \sigma_{n} \pi_{n}
\end{array} .\right.
$$

In order to solve (4.3), we make an ansatz for the Dirac operator:

$$
\begin{aligned}
\tilde{D} & \equiv D_{0}+\left(1+\mathcal{L}\left(D_{0}\right)\right) X\left(1+\mathcal{R}\left(D_{0}\right)\right) \\
& =D+X \\
D & =D_{0}+\mathcal{L}\left(D_{0}\right) X+X \mathcal{R}\left(D_{0}\right)+\mathcal{L}\left(D_{0}\right) X \mathcal{R}\left(D_{0}\right)
\end{aligned}
$$

where the $D_{0}$ is the Dirac operator in the free theory. It satisfies the original GW relation,

$$
\left\{\gamma_{5}, D_{0}\right\}=\frac{2}{\alpha} D_{0} \gamma_{5} D_{0}
$$

Let us suppose that a solution for $D_{0}$ such as the Neuberger's type [2] is given. The functions $\mathcal{L}\left(D_{0}\right)$ and $\mathcal{R}\left(D_{0}\right)$ can be fixed by substituting (4.5) into (4.3) and using (4.4) for $\delta X$. After a little algebra, we find that the Dirac operator,

$$
\tilde{D}=D_{0}+\left(1-\frac{1}{\alpha} D_{0}\right) X\left(1-\frac{1}{\alpha} D_{0} \frac{1-\frac{2}{\alpha} \gamma_{5} D_{0} \gamma_{5}}{1-\frac{1}{\alpha} \gamma_{5} D_{0} \gamma_{5}}\right)
$$

\footnotetext{
${ }^{1}$ The matrix $\gamma_{5}$ satisfies $\gamma_{5}^{2}=1$.
} 
solves the GW relation (4.1).

Let us determine the potential for the auxiliary fields $A_{X}[\pi, \sigma]$. We first consider (3.11a) to find a potential term $A_{X}^{(0)}[\pi, \sigma]$ which is invariant under the nonlinear chiral transformations (4.4) for $\pi$ and $\sigma$. It turns out to be

$$
A_{X}^{(0)}[\pi, \sigma]=\sum_{n} h\left(\frac{\pi_{n}^{2}+\sigma_{n}^{2}}{1-\frac{2 \sigma_{n}}{\alpha}+\frac{\pi_{n}^{2}+\sigma_{n}^{2}}{\alpha^{2}}}\right),
$$

where $h(x)$ is a function of $x$.

We next consider $(3.11 \mathrm{~d})$ to fix the counter action $A_{X}^{\text {count }}=A_{X}^{(1)}+A_{X}^{(2)}$. These obey

$$
\begin{aligned}
\delta A_{X}^{(1)}[\pi, \sigma] & =\delta J_{X}[\pi, \sigma]=-\sum_{n}\left(\frac{8}{\alpha} \pi_{n}\right) \\
& =i \frac{2^{2-\frac{d}{2}}}{\alpha} \sum_{n} \operatorname{Tr} \gamma_{5}\left(X-X^{\dagger}\right), \\
\delta A_{X}^{(2)}[\pi, \sigma] & =-2 i \sum_{n} \operatorname{Tr}\left(\gamma_{5}-\gamma_{5} \frac{\tilde{D}}{\alpha}\right)_{n n} \\
& =i \frac{2}{\alpha} \sum_{n} \operatorname{Tr}\left(\gamma_{5}(1+\mathcal{L}) X(1+\mathcal{R})\right)_{n n},
\end{aligned}
$$

where the trace $\operatorname{Tr}$ is taken over the spinor space. Solving these, we find

$$
\begin{aligned}
A_{X}^{(1)}[\pi, \sigma] & =\sum_{n} 2 \ln \left(1-\frac{2 \sigma_{n}}{\alpha}+\frac{\pi_{n}^{2}+\sigma_{n}^{2}}{\alpha^{2}}\right) \\
& =2^{1-\frac{d}{2}} \sum_{n} \operatorname{Tr} \ln \left(\left(1-\frac{X_{n}}{\alpha}\right)\left(1-\frac{X_{n}^{\dagger}}{\alpha}\right)\right), \\
A_{X}^{(2)}[\pi, \sigma] & =\sum_{n} \operatorname{Tr}\left(\gamma_{5}(1+\mathcal{L}) \gamma_{5} \ln \left(1-\frac{X}{\alpha}\right) \cdot(1+\mathcal{R})\right)_{n n} .
\end{aligned}
$$

We summarize here the structure of the chiral symmetry in the macroscopic theory. We may define the chiral transformation 2 as

$$
\begin{aligned}
& \delta \Psi_{n}=i \gamma_{5}\left(1-\frac{2}{\alpha} \tilde{D}\right)_{n m} \Psi_{m} \\
& \delta \bar{\Psi}_{n}=\bar{\Psi}_{n} i \gamma_{5} \\
& \delta X_{n}=-2 i \gamma_{5}\left(X_{n}-\frac{1}{\alpha} X_{n} X_{n}\right) .
\end{aligned}
$$

In the path-integral over the macroscopic fields,

$$
\begin{aligned}
& Z_{\mathrm{MACRO}} \\
& \quad=\int\left[\mathcal{D} \Psi \mathcal{D} \bar{\Psi} \mathcal{D} \pi \mathcal{D} \sigma e^{-A_{X}^{(1)}[\pi, \sigma]-A_{X}^{(2)}[\pi, \sigma]}\right] e^{-\sum_{n m} \bar{\Psi}_{n} \tilde{D}(\pi, \sigma)_{n m} \Psi_{m}-A_{X}^{(0)}[\pi, \sigma]},
\end{aligned}
$$

\footnotetext{
${ }^{2}$ For the Dirac fields, the most general form of the chiral transformation may be given by $\delta \Psi=$ $i \gamma_{5}\left[1-\alpha^{-1}(2-s) \tilde{D}\right] \Psi, \quad \delta \bar{\Psi}=\bar{\Psi} i\left(1-\alpha^{-1} s \tilde{D}\right) \gamma_{5}$, where $s$ is a real constant. In (4.13), we take $s=0$.
} 
the measure $\mathcal{D} \Psi \mathcal{D} \bar{\Psi} \mathcal{D} \pi \mathcal{D} \sigma$ multiplied by the exponential of the counter actions $e^{-\left(A_{X}^{(1)}+A_{\text {aux }}^{(2)}\right)}$ becomes invariant under (4.13). The remaining action

$$
A^{\mathrm{inv}}=\sum_{n m} \bar{\Psi}_{n}\left(D_{0}+(1+\mathcal{L})\left(i \gamma_{5} \pi+\sigma\right)(1+\mathcal{R})\right)_{n m} \Psi_{m}+A_{X}^{(0)}[\pi, \sigma]
$$

is also chiral invariant: $\delta A^{\text {inv }}=0$. In summary, we construct an exact chiral symmetry in quantum system (4.14) with the auxiliary fields describing the scalar and pseudo-scalar couplings of the fermionic self-interactions.

\section{Summary and discussion}

In this letter, we have constructed an exact chiral symmetry in the quantum system (4.14) on the coarse lattice with the auxiliary fields describing the scalar and pseudoscalar couplings of the fermionic self-interactions. The GW relation plays a key rôle in this construction, as in the case of chiral gauge theories. It should be remarked, however, that the GW relation obtained here and the one for chiral gauge theories have different structure: Although the Dirac operator in the latter depends on the gauge field, its expression takes the same form as that for free theories. In contrast to this, our GW relation (4.1) has additional contribution from the auxiliary fields. We have solved the relation under the locality assumption on the auxiliary fields. The solutions are obtained in a closed form, and used to construct the chiral transformations of the Dirac as well as the auxiliary fields on the coarse lattice. We have also solved other WT identities to fix the auxiliary-field sector of the macroscopic action, i.e., the invariant action and the counter action needed to define chiral invariant measure. In this way we have obtained chiral symmetric partition function (4.14).

The lattice chiral symmetry discussed here has yet some peculiar properties. All the fields on the coarse lattice transform nonlinearly. The $\delta \Psi$ and $\delta \bar{\Psi}$ depend on $X$, and $\delta X$ has $X^{2}$ term. As a result, none of the kinetic term $\bar{\Psi} D_{0} \Psi$, the Yukawa coupling term $\bar{\Psi}(1+\mathcal{L})\left(i \gamma_{5} \pi+\sigma\right)(1+\mathcal{R}) \Psi$, the functional measure $\mathcal{D} \Psi \mathcal{D} \Psi \mathcal{D} \pi \mathcal{D} \sigma$ becomes chiral invariant. Furthermore, the invariant operators made out of the auxiliary fields are nonpolynomial, as seen in (4.8). It makes the integration of the auxiliary fields difficult. These problems are related to the choice of the dynamical variables in our system. In a forthcoming paper [15], we describe our construction of the symmetry in more detail, and discuss the above problems as well as the structure of our lattice chiral symmetry.

\section{Acknowledgments}

This work is supported in part by the Grants-in-Aid for Scientific Research No. 12640258, 12640259, and 13135209 from the Japan Society for the Promotion of Science. 


\section{References}

[1] P. H. Ginsparg and K. G. Wilson, Phys. Rev. D25 (1982) 2649.

[2] H. Neuberger, Phys. Lett. B417 (1998) 141; Phys. Lett. B427 (1998) 353.

[3] P. Hasenfratz, V. Laniena and F. Nidermayer, Phys. Lett. B427 (1998) 125.

[4] M. Lüscher, Phys. Lett. B428 (1998) 342.

[5] P. Hasenfratz, Nucl. Phys. B525 (1998) 401.

[6] P. Hernandez, K. Jansen and M. Lüscher, Nucl. Phys. B552 (1999) 363.

[7] H. Neuberger, Phys. Rev. D57 (1998) 5417.

[8] I. Horvath, Phys. Rev. Lett. 81 (1998) 4063.

[9] H. B. Nielsen and M. Ninomiya, Nucl. Phys. B185 (1981) 20; [E: B195 (1982) 541]; B193 (1981) 173.

[10] F. Nidermayer, Nucl. Phys. Proc. Supple. 73 (1999) 105.

H. Neuberger, "Exact chiral symmetry on the lattice", hep-lat/0101006.

M. Lüscher, "Chiral gauge theories revised", hep-th/0102028.

M. Creutz, "Aspects of chiral symmetry and the lattice", hep-lat/0007032.

[11] M. Lüscher, Nucl. Phys. B538 (1999) 515; Nucl. Phys. B549 (1999) 295.

[12] T. W. Chiu, C. W. Wang and S. V. Zenkin, Phys. Lett. B438 (1998) 321.

[13] I. Ichinose and K. Nagao, Chin. J. Phys. 38 (2000) 671; hep-lat/9909035; Mod. Phys. Lett. A15 (2000) 857.

[14] Y. Igarashi, K. Itoh and H. So, Phys. Lett. B526 (2002) 164.

[15] Y. Igarashi, H. So and N. Ukita, in preparation. 